\title{
What geometrical factors determine the in situ solar wind speed?
}

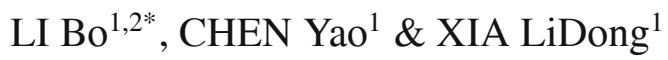 \\ ${ }^{1}$ Shandong Provincial Key Laboratory of Optical Astronomy \& Solar-Terrestrial Environment, School of Space Science and Physics, Shandong \\ University at Weihai, Weihai 264209, China; \\ ${ }^{2}$ State Key Laboratory of Space Weather, Chinese Academy of Sciences, Beijing 100190, China
}

Received September 6, 2011; accepted November 22, 2011; published online February 21, 2012

\begin{abstract}
At present it remains to address why the fast solar wind is fast and the slow wind is slow. Recently we have shown that the field line curvature may substantially influence the wind speed $v$, thereby offering an explanation for the Arge et al. finding that $v$ depends on more than just the flow tube expansion factor. Here we show by extensive numerical examples that the correlation between $v$ and field line curvature is valid for rather general base boundary conditions and for rather general heating functions. Furthermore, the effect of field line curvature is even more pronounced when the proton-alpha particle speed difference is examined. We suggest that any solar wind model has to take into account the field line shape for any quantitative analysis to be made.
\end{abstract}

solar wind, magnetic field, Magnetohydrodynamic waves, space weather

Citation: Li B, Chen Y, Xia L D. What geometrical factors determine the in situ solar wind speed? Chin Sci Bull, 2012, 57: 1409-1414, doi: 10.1007/s11434-011-4965-2

Half a century after its prediction [1] and direct detection [2], how the solar wind is accelerated to its highly supersonic terminal speed remains elusive. Among the extensive experimental findings that provide stringent constraints on these mechanisms, those concerning the in situ wind speed $v$ are of primary concern from both theoretical and practical standpoints: $v$ and the magnetic field polarity near the Earth are the two main parameters involved for predicting geomagnetic activities, a major player in space weather [3-6]. A widely used technique to predict $v$ is the Wang-Sheeley-Arge model [7], which is based on two well-established statistical relations between $v$ and the properties of the flow tube $\mathcal{L}$ along which the wind flows. First, $v$ is inversely correlated with the coronal expansion rate $f_{c}$ of $\mathcal{L}$ [8]. Second, for a given $f_{c}, v$ is inversely correlated with the angular distance $\theta_{b}$ of the footpoint of $\mathcal{L}$ to its nearest coronal hole boundary [7]. Given the importance of the wind speed prediction, the two results deserve a sound physical explanation. While the former is well supported by a number of wave/turbulence-based solar wind models (see [9] and references therein), the latter has so far attracted little attention.

*Corresponding author (email: bbl@ sdu.edu.cn)
Close inspections of coronal images indicate that the further away the field line footpoint is from the coronal hole boundary, the more curved the field line is (see e.g. the composite image Figure 1 in [10]). Consequently, the effects of $\theta_{b}$ on the wind speed may be explained in terms of field line curvature. A detailed modeling study using a sophisticated Alfvénic-turbulence-based heating mechanism suggests that it is indeed so ([11], hereafter paper I). The model distinguishes between electrons and protons, adopts reasonably complete energy equations including radiative losses, electron heat conduction, and solar wind heating. It also employs the Radiation Equilibrium Boundary (REB) at the base which is set at the mid-Transition region, whereby the base electron density automatically adjusts to the downward heat flux from the corona $[12,13]$. Compared with a straight flow tube with identical radial distribution of the magnetic field strength, a curved tube produces a significantly reduced flow speed at $1 \mathrm{AU}$ (by up to $\sim 30 \%$ ), accompanied by an enhanced mass flux density. This effect was interpreted, in view of the general framework by [14], as a result of the enhanced energy deposition in the near-Sun region in the curved case relative to the straight one since the elementary reduction in the wave action flux density is a factor of $\mathrm{d} l / \mathrm{d} r$ larger (eq. (7) in paper 
I), where $l$ is the arclength and $r$ the heliocentric distance.

The aim of the present paper is to extend paper I by examining whether the effect of field line curvature discovered there is robust enough: Is it valid only for this particular boundary condition or this particular heating mechanism? Will the inclusion of alpha particles, the second most abundant ion species, invalidate the conclusion? The latter is of particular importance since alpha particles, with their mass being 4 times the proton one, may play an important role in regulating the wind parameters [15-17].

\section{Model description}

Let us start with considering a time-independent solar wind that consists of electrons $(e)$, protons $(p)$, and alpha particles $(\alpha)$. Each species $s(s=e, p, \alpha)$ is characterized by its mass $m_{s}$, electric charge $e_{s}$, number density $n_{s}$, mass density $\rho_{s}=n_{s} m_{s}$, velocity $\boldsymbol{v}_{s}$, temperature $T_{s}$ and partial pressure $p_{s}=n_{s} k_{B} T_{s}$. Here $k_{B}$ is the Boltzmann constant. We may express $e_{s}$ in units of the electron charge $e$, i.e. $e_{s}=Z_{s} e$ with $Z_{e} \equiv-1$ by definition. Assuming axisymmetry, quasineutrality and quasi-zero current, neglecting solar rotation, one may readily decompose the vector equations in the standard 5-moment treatment into a force balance equation across the meridional magnetic field $\boldsymbol{B}$ and a set of transport equations along it (for a formal development, see section 2.1 in [18]). This transport equation set reads

$$
\begin{aligned}
& B\left(\frac{n_{k} v_{k}}{B}\right)^{\prime}=0, \\
& v_{k} v_{k}^{\prime}+\frac{p_{k}^{\prime}}{n_{k} m_{k}}+\frac{Z_{k} p_{e}^{\prime}}{n_{e} m_{k}}+\frac{G M_{\odot}}{r}(\ln r)^{\prime} \\
& -\frac{1}{n_{k} m_{k}}\left(\frac{\delta M_{k}}{\delta t}+\frac{Z_{k} n_{k}}{n_{e}} \frac{\delta M_{e}}{\delta t}\right)=a_{k}, \\
& v_{s}\left(\frac{p_{s}}{\gamma-1}\right)^{\prime}+\frac{\gamma p_{s}}{\gamma-1} B\left(\frac{v_{s}}{B}\right)^{\prime} \\
& +B\left(\frac{q_{s}}{B}\right)^{\prime}-\frac{\delta E_{s}}{\delta t}-Q_{s}+L=0,
\end{aligned}
$$

where the subscript $s$ refers to all species $(s=e, p, \alpha)$, while $k$ stands for ion species only $(k=p, \alpha)$. The gravitational constant is denoted by $G, M_{\odot}$ is the mass of the Sun. The prime " "' denotes the derivative with respect to the arclength $l$, measured from the footpoint of a magnetic field line. The momentum and energy exchange rates due to the Coulomb collisions of species $s$ with the remaining ones are denoted by $\delta M_{s} / \delta t$ and $\delta E_{s} / \delta t$, respectively. Their expressions may be found in [19] for which we use a Coulomb logarithm $\ln \Lambda=23$. As for $a_{k}$, it represents the acceleration exerted on ion species $k$. Moreover, $q_{s}$ is the heat flux carried by species $s$, and $Q_{s}$ stands for the heating rate applied to species $s$ from non-thermal processes. $L$ is the radiative loss function involved in the electron energy equation only. It is assumed to be optically thin and follows the analytic form given in [20]. To simplify our treatment, we neglect ion heat fluxes $\left(q_{k}=0\right)$, and adopt the Spitzer law for the electron one $q_{e}=-\kappa T_{e}^{5 / 2} T_{e}^{\prime}$ where $\kappa=7.8 \times 10^{-7}$ erg $\mathrm{K}^{-7 / 2} \mathrm{~cm}^{-1} \mathrm{~s}^{-1}$ [21].

As detailed in paper I, rather than solving for a fully selfconsistent multi-dimensional solution, we solve eqs. (1) to (3) on a prescribed magnetic field instead, the reason being that in a multi-dimensional model the effects of magnetic field expansion and field line curvature cannot be distinguished. In practice, we employ the analytic model given by [22] for which the reference values $\left[Q, K, a_{1}\right]=$ $[1.5,1,1.538]$ are used. The resulting magnetic field configuration agrees favorably with the coronal images obtained with SOHO/LASCO [23], and has been widely seen as being representative of a minimum solar corona. In line with the Ulysses measurements, the magnetic field strength at $1 \mathrm{AU}$ is essentially latitude-independent and is $3.5 \gamma$ [24,25]. All the presented results will be computed along the field line that reaches $1 \mathrm{AU}$ at $88^{\circ}$ colatitude, nearly maximizing the effect of field line curvature.

Specifying the boundary condition (BC) at the base $r=$ $1 R_{\odot}$ is crucial for constructing wind solutions. We will test three BCs: REB, TR, and CB. By REB, we mean the Radiation Equilibrium Boundary where a base temperature $T_{s 0}=5 \times 10^{5} \mathrm{~K}$ is set, the electron density $n_{e 0}=4.8 \times 10^{3} q_{e 0}$, in which the subscript " 0 " represents the base value $[12,13]$. Besides, TR (CB) refers to the case where we set the base at the lower Transition-Region (coronal base), $T_{s 0}=10^{5} \mathrm{~K}$ $\left(10^{6} \mathrm{~K}\right)$, the corresponding $n_{e 0}$ being $10^{9}\left(1.5 \times 10^{8}\right) \mathrm{cm}^{-3}$.

The source terms $a_{k}$ and $Q_{s}$ remain to be specified. In our study they depend on whether alpha particles are included, and on how the base BC is implemented. Electron heating is applied for the REB and TR boundaries to compensate for the significant radiative losses at the corresponding temperatures. It is in the form $Q_{e}=Q_{e 0} \exp \left(-l / l_{d}\right)$, with $Q_{e 0}$ (in $10^{-5} \mathrm{erg} \mathrm{cm}^{-3} \mathrm{~g}^{-1}$ ) and $l_{d}$ (in $R_{\odot}$ ) being 1.7 and 0.06 for REB, 2.9 and 0.06 for TR. For ion heating, either the wave heating scenario or some empirical heating function is employed. The former choice is used only in the absence of alpha particles. In this case a wave transport equation (eq. (4) in paper I) is included where the parallel propagating Alfven waves lose their energy via both doing work on the flow through the ponderomotive force ( $a_{k}$, see eq. (2) in paper I) and dissipation at the Kolmogorov rate. For more recent developments on the role of Magnetohydrodynamic waves in energizing plasmas, see e.g. [26]. We use a base wave amplitude of 27,18 , and $29 \mathrm{~km} \mathrm{~s}^{-1}$, a base value of correlation length being $1.5,2$, and $2 \times 10^{4} \mathrm{~km}$, for the REB, TR and CB boundaries, respectively. These parameters are within observational limits, and produce solar wind solutions whose terminal speed and proton flux agree with in situ measurements. When ions are heated in an empirical fashion, we assume the external energy comes from an ad hoc energy flux dissipated at a constant length $l_{\mathrm{ad}}$, the resulting total heating rate being $Q=F_{E} B /\left(B_{E} l_{\mathrm{ad}}\right) \exp \left(-l / l_{\mathrm{ad}}\right)$. Here $F_{E}$ is the input flux scaled to the Earth orbit $r_{E}=1 \mathrm{AU}$, and $B_{E}$ is the meridional magnetic field strength at $r_{E} . Q$ is apportioned between pro- 
tons and alpha particles, and some energy that alpha particles receive turns out necessary to be in the form of momentum deposition:

$$
\begin{aligned}
& Q_{p}+\bar{Q}_{\alpha}=Q, \frac{\bar{Q}_{\alpha}}{Q_{p}}=\chi_{E} \frac{\rho_{\alpha}}{\rho_{p}}, \\
& Q_{\alpha}+\rho_{\alpha} v_{\alpha} a_{\alpha}=\bar{Q}_{\alpha}, \frac{a_{\alpha}}{Q_{\alpha}}=\frac{\chi_{D}}{\rho_{\alpha} v_{0}},
\end{aligned}
$$

where $v_{0}=\sqrt{k_{B} T_{p} / m_{p}}$ is some characteristic speed. The momentum deposition to protons is neglected. This way for empirically heating the ion fluids seems rather involved, but actually it manages to capture some essential features of wave heating in a multifluid treatment (for details, see [27], section 3.1). In practice, we use $F_{e}=1.4 \mathrm{erg} \mathrm{cm}^{-2} \mathrm{~s}^{-1}$ and $l_{\mathrm{ad}}=1.75 R_{\odot}$, regardless of the presence of alpha particles, such that the resulting proton flux and speed at $1 \mathrm{AU}$ agree with the in situ measurements. When alpha particles exist, we use $\chi_{E}=6$ and $\chi_{D}=2$, the purpose being to generate a wind profile that corresponds to a positive proton-alpha speed difference (see section 2 for further details.)

Table 1 summarizes our computations. The first column indicates in which section the combinations of BCs and ion/electron heating in the same row are tested. For boundary conditions imposed at the base, REB (Radiation Equilibrium Boundary), TR (Transition Region), and CB (Coronal Base) conditions are experimented with. For electron heating, $\mathrm{Y}(\mathrm{N})$ refers to the case where the heating is switched on (off). As for the ion heating, "wave" and "empirical" represent the case where the ions are heated by turbulent Alfvén waves and in an empirical fashion, respectively. The last column indicates whether or not alpha particles are included in the computations, with $\mathrm{Y}$ and $\mathrm{N}$ meaning that they are and are not, respectively.

\section{Numerical results}

Now we are in a position to examine how robust the effects of field line curvature are by varying our choices of base BC, the proton heating, and the inclusion of alpha particles. For any combination of the three choices, we compute two solar wind solutions that differ only in whether or not the field line curvature is considered. If not (yes), the solution is labeled $\mathrm{S}(\mathrm{C})$, short for straight (curved). Before proceeding, let us briefly discuss what we mean by "curvature". Similar to paper I, here it is defined in an intuitive rather than a precise manner, and refers to how significantly the field line differs from a radial shape. Locally speaking, at any given point along a field line, the curvature can be precisely defined, say, in terms of the curvature radius $\mathcal{R}$. However, the effect of field line shape on the wind parameters turns out to be a cumulative one to which the shape at each and every point contributes. A nonlocal definition of the field line shape by, say, integrating $1 / \mathcal{R}$ is for sure more precise, but evaluating its correlation with the computed wind parameters is beyond the scope of the present paper.

\subsection{Influences of the base boundary condition (without alpha particles)}

Let us for now neglect alpha particles but focus on the influence of the boundary condition imposed at the base. Figure 1 presents the distribution of the proton speed $v_{p}$ with the heliocentric distance $r$. It compares the solutions obtained in the curved cases (solid lines) with those in the straight cases (dashed lines). The black, red, and green curves are for REB, TR, and CB, respectively. Moreover, the vertical bars represent wind speeds measured by tracking small density inhomogeneities (blobs) in SOHO/LASCO images as given

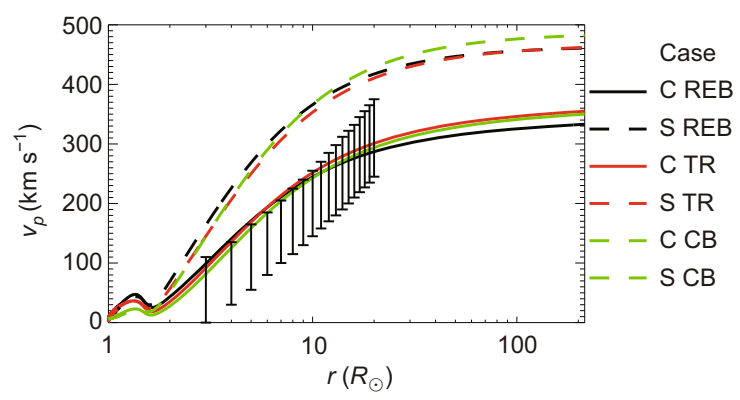

Figure 1 Effects of field line curvature on wind speed under the influence of different base boundary conditions. Here the proton speed $v_{p}$ is shown as a function of heliocentric distance $r$. The solid (dashed) curves are for the solutions that incorporate (neglect) the field line curvature. Black, red, and green curves represent solutions using the REB, TR, and CB boundaries, respectively. Moreover, the vertical bars represent wind speeds measured by tracking small density inhomogeneities (blobs) in SOHO/LASCO images as given by [28].

\begin{tabular}{|c|c|c|c|c|c|c|c|c|c|}
\hline \multirow{2}{*}{ Section } & \multicolumn{3}{|c|}{ Base boundary condition } & \multicolumn{2}{|c|}{$e^{-}$heating } & \multicolumn{2}{|c|}{ Ion heating } & \multicolumn{2}{|c|}{$\alpha$-particles included? } \\
\hline & REB & TR & $\mathrm{CB}$ & $\mathrm{Y}$ & $\mathrm{N}$ & Wave & Empirical & $\mathrm{Y}$ & $\mathrm{N}$ \\
\hline \multirow[t]{3}{*}{2.1} & $\checkmark$ & & & $\checkmark$ & & $\checkmark$ & & & $\checkmark$ \\
\hline & & $\checkmark$ & & $\checkmark$ & & $\checkmark$ & & & $\checkmark$ \\
\hline & & & $\checkmark$ & & $\checkmark$ & $\checkmark$ & & & $\checkmark$ \\
\hline 2.3 & & & $\checkmark$ & & $\checkmark$ & & $\checkmark$ & $\checkmark$ & \\
\hline
\end{tabular}

Table 1 Combinations of base boundary conditions, electron and proton heating, and inclusion of alpha particles 
by [28]. One can see that all lines, both solid and dashed, possess a dip below $2 R_{\odot}$, indicating that this local minimum in $v_{p}$ results from the drastic, lateral expansion of the flow tube rather than its curvature. On the other hand, all the solid curves reproduce the SOHO/LASCO measurements equally well, and they reach similar values at $1 \mathrm{AU}$. To be specific, the REB, TR, and CB solutions yield a $v_{p}$ of 333, 355, and $351 \mathrm{~km} \mathrm{~s}^{-1}$, respectively. The corresponding values in solutions with identical BCs but neglecting field line curvature are 461, 462, and $482 \mathrm{~km} \mathrm{~s}^{-1}$ respectively. Moreover, the proton flux at $1 \mathrm{AU}$ (in $10^{8} \mathrm{~cm}^{2} \mathrm{~s}^{-1}$ ) is found to be 3.65(3.14) in the curved (straight) case for REB, 3.86 (3.47) for TR, 3.57 (3) for CB. In other words, one finds a reduction in $v_{p}$, relative to the straight case, of $27.8 \%$ for REB, $23.2 \%$ for TR, and $27.4 \%$ for $\mathrm{CB}$, and an enhancement in proton flux by $16.1 \%$ for REB, $11.4 \%$ for TR, and $18.9 \%$ for $\mathrm{CB}$, respectively. Hence, the significant reduction in the terminal proton speed and enhancement in the proton flux due to the inclusion of field line curvature is not specific to the particular REB condition employed in paper I, but happens for rather general boundary conditions.

\subsection{Influences of proton heating}

Is the effect of field line curvature present only for the particular ion heating mechanism used in paper I? This is examined in Figure 2 where we again neglect alpha particles but do employ a different ion heating function, namely the empirical heating rates detailed in section 1 . Figure 2 presents the radial distributions of the proton speed $v_{p}$, for both cases $\mathrm{C}$ (solid lines) and $\mathrm{S}$ (dashed lines). Comparison of Figure 2 with Figure 1 indicates that the proton speed profile in Case $\mathrm{C}$ is steeper than in the wave-driven model, nonetheless the effect of field line curvature remains the same. To be specific, the terminal speed is reduced from 583 in the straight to $399 \mathrm{~km} \mathrm{~s}^{-1}$ in the curved case. Likewise, the proton flux (in $10^{8} \mathrm{~cm}^{2} \mathrm{~s}^{-1}$ ) increases from 2.61 to 3.47 . This change in the proton parameters can be readily explained using the same argument as in the wave-driven case. The energy deposition

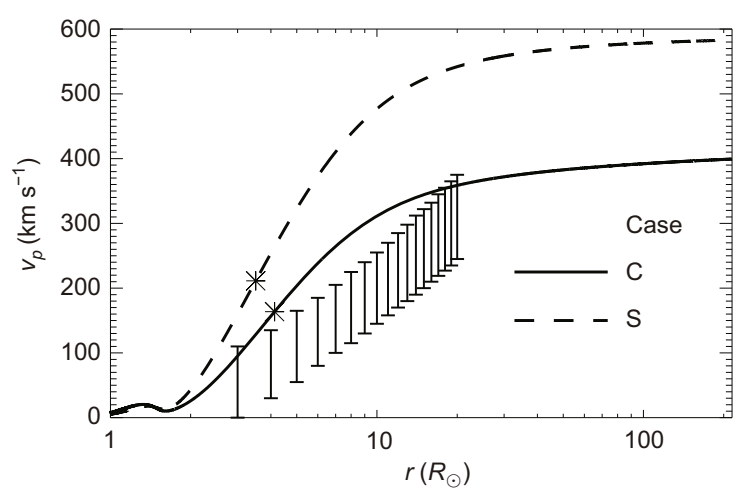

Figure 2 Effects of field line curvature on the proton speed for an empirically heated wind. The solid (dashed) curves are for the solutions that incorporate (neglect) the field line curvature. The vertical bars have the same meaning as in Figure 1. As for the asterisks, they represent the critical points where the proton speed equals the sound speed (see text for details). in the subsonic region can be expressed as an energy flux density scaled to $1 \mathrm{AU}, F_{\text {sub }}=\int_{R_{\odot}}^{r_{C}} Q B_{E} / B(\mathrm{~d} l / \mathrm{d} r) \mathrm{d} r$, evaluating which results in $F_{E}\left[1-\exp \left(-l_{C} / l_{\text {ad }}\right)\right]$. Here the subscript $C$ represents the values at the critical point where $v_{p}$ equals $\sqrt{k_{B}\left(T_{e}+T_{p}\right) / m_{p}}$. It follows that if in Cases $\mathrm{C}$ and $\mathrm{S}$ the critical point lies at the same radial distance, $F_{\text {sub }}$ is larger in Case $\mathrm{C}$ given that $l_{C}$ is larger. And from the general framework presented in [14] it can be readily deduced that Case C will yield an enhanced proton flux and a reduced proton terminal speed. Actually this effect is compounded by the fact that in Case $\mathrm{C}$ the critical point is located further away from the Sun: $r_{C}=4.13$ and $3.51 R_{\odot}$ in Cases $\mathrm{C}$ and $\mathrm{S}$, respectively.

\subsection{Influences of alpha particles}

Finally let us address the role of alpha particles. For simplicity we employ the empirical ion heating rates, since we have already shown that adopting some more sophisticated mechanism like wave heating will not change our result as far as the effect of field line curvature is concerned. Figure 3 examines how the flow parameters at $1 \mathrm{AU}$ change with varying $n_{\alpha p, 0}$, the alpha abundance at the coronal base. These include the proton speed $v_{p, E}$, proton flux density $\left(n_{p} v_{p}\right)_{E}$, and proton-alpha speed difference $v_{\alpha p, E}=\left(v_{\alpha}-v_{p}\right)_{E}$. The solid and dashed curves are for Cases $C$ and $S$, respectively. Figure 3(a) indicates that for $n_{\alpha p, 0}$ in the range from $3 \times 10^{-5}$ to 0.1 , the field line curvature consistently reduces the terminal proton speed and enhances the proton flux density. For $n_{\alpha p, 0} \lesssim 10^{-2}$, introducing alpha particles has little effect
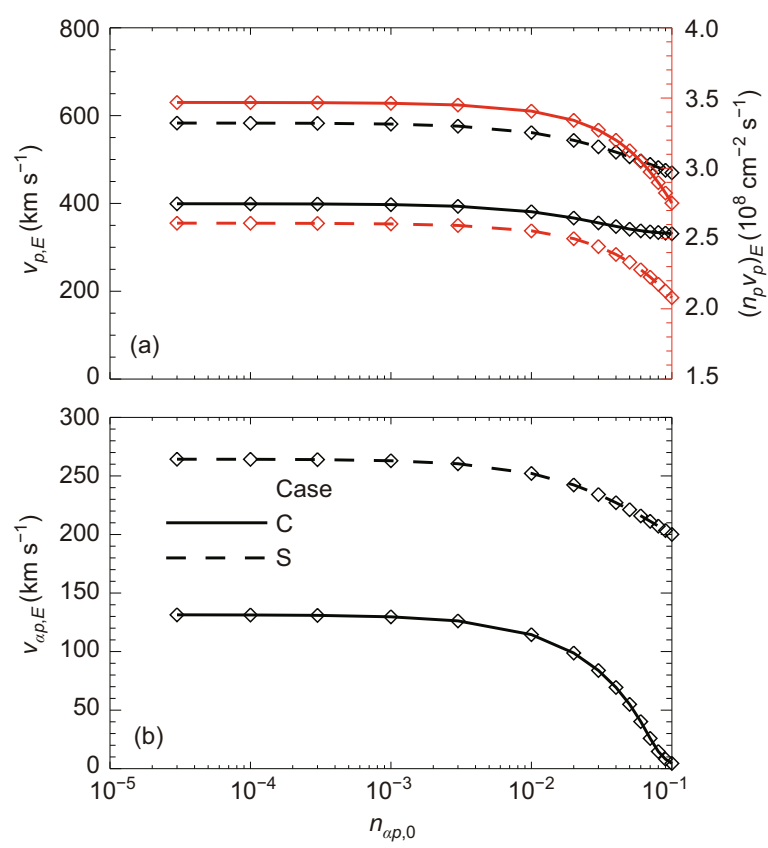

Figure 3 Effects of field line curvature on flow parameters for an electronproton-alpha wind. Displayed as a function of the base alpha abundance $n_{\alpha p, 0}=\left(n_{\alpha} / n_{p}\right)_{0}$ are values at $1 \mathrm{AU}$ of (a) the proton speed $v_{p, E}$ and proton flux density $\left(n_{p} v_{p}\right)_{E}$, and (b) the proton-alpha speed difference $v_{\alpha p, E}=$ $\left(v_{\alpha}-v_{p}\right)_{E}$. The solid (dashed) curves are for the solutions that incorporate (neglect) the field line curvature. 
on the proton parameters and the reduction in $v_{p, E}$ by the field line curvatures is $\sim 183 \mathrm{~km} \mathrm{~s}^{-1}$, or by $\sim 31.5 \%$. On the other hand, for $n_{\alpha p, 0}=0.1$, Case C yields a $v_{p, E}$ of $331 \mathrm{~km} \mathrm{~s}^{-1}$, or down by $29.6 \%$ relative to Case $\mathrm{S}$ where $v_{p, E}$ is $470 \mathrm{~km} \mathrm{~s}^{-1}$. Hence whether or not the alpha particles are included, introducing field line curvature has the same effect on the proton parameters in interplanetary space.

The proton-alpha speed difference $v_{\alpha p}$ has been a subject of extensive study (e.g. [29,30] and references therein). Figure 3(b) shows that the field line curvature significantly reduces $v_{\alpha p}$. This is true throughout the range of $n_{\alpha p, 0}$ explored. For instance, the amount of reduction in $v_{\alpha p, E}$ is $133 \mathrm{~km} \mathrm{~s}^{-1}$ for $n_{\alpha p, 0}=3 \times 10^{-5}$ (from 264.3 in Case $\mathrm{S}$ to $131.4 \mathrm{~km} \mathrm{~s}^{-1}$ in Case C), and is $195.5 \mathrm{~km} \mathrm{~s}^{-1}$ for $n_{\alpha p, 0}=0.1$ (from 200 in Case $\mathrm{S}$ to $4.54 \mathrm{~km} \mathrm{~s}^{-1}$ in Case $\mathrm{C}$ ). This significant reduction in $v_{\alpha p}$ at large distances turns out to be reminiscent of what happens in the corona: although not shown, the alpha temperature is substantially higher in Case $S$ than in Case C from $1.5 R_{\odot}$ onward, resulting in a substantially larger alpha pressure gradient force and hence a significantly larger proton-alpha speed difference. From this we may conclude that, the effect of field line curvature should be taken into account for a quantitative investigation into the low-latitude slow wind, and its role in regulating the ion speed difference is even more pronounced. Once this effect is introduced, one may expect to see some significant revision to some recent studies (e.g. [17,31]), where the flow tube is taken to be radially directed.

\section{Conclusions}

The present study has been motivated by the fact that for space weather applications, the near-Earth solar wind speed $v$ is of crucial importance and is being routinely predicted by the Wang-Sheeley-Arge (WSA) model [7]. This model is based on the relations between $v$ and the coronal expansion rate of the solar wind flow tube [8] as well as the angular distance of the tube footpoint to its nearest coronal hole boundary [7]. While the former correlation has been extensively examined, the latter has been given little physical interpretation, and was only recently explained in terms of the field line curvature [11]: the more curved the flow tube (or equivalently the magnetic field line) is, the higher the proton flux density, and the lower the terminal proton speed. Given that the conclusion was established only via a limited number of computations, and given that providing a sound physical explanation for the WSA model puts considerably more confidence in this model, we show in this paper by extensive numerical examples that the correlation between the terminal proton speed and field line curvature is valid for rather general base boundary conditions and for rather general heating functions commonly adopted in the literature. Furthermore, its validity is not violated by the introduction of alpha particles, the second most abundant ion species in the corona and solar wind. An immediate consequence of our new computations is that any solar wind model, for the fast and slow wind alike, has to take into account the field line shape for any quantitative analysis to be made. Besides, the effect of field line curvature is even more pronounced when examining the proton-alpha particle speed difference, thereby calling for the urgent inclusion of this effect in multi-fluid models of the low-latitude solar winds where the ion speed difference is among the primary concerns.

This work was supported by the National Natural Science Foundation of China (40904047, 40825014, 40890162 and 40974105) and the Specialized Research Fund for State Key Laboratories.

1 Parker E N. Dynamics of the interplanetary gas and magnetic fields. Astrophys J, 1958, 128: 664-676

2 Neugebauer M, Snyder C W. Solar plasma experiment. Science, 1962, 138: 1095-1097

3 Zhao H, Zong Q G, Wei Y, et al. Influence of solar wind dynamic pressure on geomagnetic $D_{\text {st }}$ index during various magnetic storms. Sci China Tech Sci, 2011, 54: 1445-1454

4 Wei Y, Zong Q G, Pu Z Y, et al. Enhanced anti-sunward flow near local noon during a period of horizontal IMF and high solar wind velocity VY. Chin Sci Bull, 2011, 56: 1117-1122

5 Zong Q G, Wang Y F, Yuan C J, et al. Fast acceleration of killer electrons and energetic ions by interplanetary shock stimulated ULF waves in the inner magnetosphere. Chin Sci Bull, 2011, 56: 1188-1201

6 Feng Y Y, Zhang Y T, Zhao H, et al. Solar wind electrons reflected by lunar electric and magnetic fields. Sci China Earth Sci, 2011, 54: 1796-1800

7 Arge C N, Luhmann J G, Odstrcil D, et al. Stream structure and coronal sources of the solar wind during the May 12th, 1997 CME. J Atmosph Solar-Terrest Phys, 2004, 66: 1295-1309

8 Wang Y-M, Sheeley N R Jr. Solar wind speed and coronal flux-tube expansion. Astrophys J, 1990, 355: 726-732

9 Cranmer S R, van Ballegooijen A A, Edgar R J. Self-consistent coronal heating and solar wind acceleration from anisotropic magnetohydrodynamic turbulence. Astrophys J Suppl Series, 2007, 171: 520-551

10 Forsyth R J, Marsch E. Solar origin and interplanetary evolution of stream interfaces. Space Sci Rev, 1999, 89: 7-20

11 Li B, Xia L D, Chen Y. Solar winds along curved magnetic field lines. Astron Astrophys, 2011, 529: A148

12 Withbroe G L. The temperature structure, mass, and energy flow in the corona and inner solar wind. Astrophys J, 1988, 325: 442-467

13 Lionello R, Linker J A, Mikić Z. Including the transition region in models of the large-scale solar corona. Astrophys J, 2001, 546: 542-551

14 Leer E, Holzer T E. Energy addition in the solar wind. J Geophys Res, 1980, 85: 4681-4688

15 Leer E, Holzer T E, Shoub E C. Solar wind from a corona with a large helium abundance. J Geophys Res, 1992, 97: 8183-8201

16 Li X, Esser R, Habbal S R, et al. Influence of heavy ions on the highspeed solar wind. J Geophys Res, 1997, 102: 17419-17432

17 Lie-Svendsen $\varnothing$, Hansteen V H, Leer E. Helium abundance in the corona and solar wind: Gyrotropic modeling from the chromosphere to 1 AU. Astrophys J, 2003, 596: 621-645

18 Li B, Li X. Propagation of non-Wentzel-Kramers-Brillouin Alfvén waves in a multicomponent solar wind with differential ion flow. Astrophys J, 2007, 661: 1222-1233

$19 \mathrm{Li} \mathrm{B,} \mathrm{Li} \mathrm{X.} \mathrm{Effects} \mathrm{of} \alpha$ particles on the angular momentum loss from the Sun. Astron Astrophys, 2006, 456: 359-365 
20 Rosner R, Tucker W H, Vaiana G S. Dynamics of the quiescent solar corona. Astrophys J, 1978, 220: 643-645

21 Spitzer Jr L. Physics of Fully Ionized Gases. New York: WileyInterSience, 1962

22 Banaszkiewicz M, Axford W I, McKenzie J F. An analytic solar magnetic field model. Astron Astrophys, 1998, 337: 940-944

23 Schwenn R, Inhester B, Plunkett S P, et al. First view of the extended green-line emission corona at solar activity minimum using the LascoC1 coronagraph on SOHO. Sol Phys, 1997, 175: 667-684

24 Smith E J, Balogh A. Ulysses observations of the radial magnetic field. Geophys Res Lett, 1995, 22: 3317-3320

25 Smith E J. Solar cycle evolution of the heliospheric magnetic field: The Ulysses legacy. J Atmosph Solar-Terrest Phys, 2011, 73: 277-289

26 Chen L, Wu D J. Exact solutions of dispersion equation for MHD waves with short-wavelength modification. Chin Sci Bull, 2011, 56: 955-961

27 Li B, Habbal S R, Li X. Angular momentum transport and proton-alphaparticle differential streaming in the solar wind. Astrophys J, 2007, 661: 593-601

28 Wang Y-M, Sheeley N R, Socker D G, et al. The dynamical nature of coronal streamers. J Geophys Res, 2000, 105: 25133-25142

29 Kaghashvili E K, Vasquez B J, Hollweg J V. Deceleration of streaming alpha particles interacting with waves and imbedded rotational discontinuities. J Geophys Res, 2003, 108: 1036

30 Araneda J A, Maneva Y, Marsch E. Preferential heating and acceleration of $\alpha$ particles by Alfvén-cyclotron waves. Phys Rev Lett, 2009, 102: 175001

31 Janse $\AA$ M, Lie-Svendsen $\varnothing$, Leer E. Solar wind originating in funnels: Fast or slow? Astron Astrophys, 2007, 474: 997-1013

Open Access This article is distributed under the terms of the Creative Commons Attribution License which permits any use, distribution, and reproduction in any medium, provided the original author(s) and source are credited. 